\title{
ON THE CONSTRUCTION OF A GENERALIZED COMPUTATIONAL EXPERIMENT IN VERIFICATION PROBLEMS
}

\author{
A. K. ALEKSEEV, A.E. BONDAREV*, V.A. GALAKTIONOV, A.E. KUVSHINNIKOV \\ Keldysh Institute of Applied Mathematics Russian Academy of Sciences \\ Moscow, Russia \\ *Corresponding author. E-mail: bond@ keldysh.ru
}

\section{DOI: 10.20948/mathmontis-2020-48-3}

Summary. This work is devoted to the application of a generalized computational experiment for a comparative assessment of numerical methods accuracy. A generalized computational experiment allows one to obtain a numerical solution for a class of problems determined by the ranges of defining parameters variation. The applications of such approach in the presence of a reference solution and in its absence are discussed. An example of error surfaces constructing is given when the solvers of the OpenFOAM software package are compared. The classic inviscid problem of oblique shock wave is used as a basic task. Variations of the key parameters of the problem - the Mach number and angle of attack are considered. In addition to the OpenFOAM solvers, the comparison included the WW method, which has a second order of accuracy in time and space and an adjustable artificial viscosity. The problem of flow around a cone at an angle of attack with varying Mach number, cone angle and angle of attack is also considered. The concept of an error index is introduced as an integral characteristic of deviations from the exact solution for each solver.

\section{INTRODUCTION}

Throughout the history of the development of computational mathematics and mathematical modeling, problems of verification of numerical methods have occupied a special place. When creating a new numerical method or modifying an existing one, the authors had to show the efficiency of their developments and evaluate their accuracy before proceeding with solving practical problems. A huge number of works are devoted to these studies. As an example, we can point to the works [1-12]. Verification of the obtained results and assessment of the accuracy of the applied numerical method was an obligatory part of research in the field of mathematical modeling of physical processes.

As a rule, a comparison of the numerical results was carried out with some reference solution, in the role of which the exact solution was used if available or the available experimental data. A separate problem is the estimation of the accuracy of numerical methods in the absence of a reference solution.

The relevance of the problems of verification of numerical methods and calculations based on them is also evidenced by the presence of federal standards, both foreign $[13,14]$ and recently appeared Russian [15]. Such standards determine the direction of research in this area. However, all these methodological documents are focused on verification in relation to a specific task with fixed values of key parameters.

2010 Mathematics Subject Classification: 49Q99, 76M27.

Key words and Phrases: generalized computational experiment, numerical methods, verification problems, flow around a cone, oblique shock wave, error surfaces, error index. 
It should be noted that at present the relevance of verification problems is steadily increasing due to the widespread use of open and commercial packages for solving various problems of mathematical modeling. As a rule, such packages provide the user with a certain set of numerical methods presented in the form of solvers integrated into the software package. In this case, the user is faced with the problem of choosing a solver. And here a number of difficulties arise. Not all solvers undergo comprehensive testing before being implemented into a software package. Commercial packages do not provide complete open information about the implemented numerical methods and their properties. Various development teams can add solvers to open source packages, but they often cannot provide full testing. Therefore, research in the field of verification and comparative evaluation of numerical methods is becoming more and more necessary.

Historically, verification in problems of computational aerogasdynamics consisted of two parts. The first is modeling a qualitative flow pattern containing discontinuities, separated flows, vortices, etc. The second is to ensure the accuracy of the calculation of quantitative characteristics. Here it was necessary to rely on a reference solution - experimental, accurate, or obtained by calculations using other methods.

Verification was usually carried out for one separate task. By default, it was assumed that with a small variation in the governing parameters of the problem (velocity, viscosity, time scales, thermophysical characteristics of the medium, geometric parameters), the numerical method under consideration will be applicable and provide a similar accuracy.

At the present stage, researchers need more comprehensive estimates of the accuracy of numerical methods. For example, the researchers need to have an assessment of accuracy, not for a single task, but for a class of tasks. By a class of tasks we mean a basic task considered in the ranges of change in the set of key parameters. In computational aerodynamics characteristic numbers that determine flow velocity, viscosity, thermophysical properties of the medium, geometric parameters, etc., can serve as such parameters. An opportunity of getting solution for a class of problems is provided by the construction of a generalized computational experiment. Also, a generalized computational experiment can be very useful in assessing the accuracy in the absence of a reference solution. In this case, it is possible to estimate the accuracy using an ensemble of solutions obtained by various numerical methods. The variation of the solver is considered as a parameter and the parametric problem is solved using a generalized computational experiment.

The concept, basic methods and approaches of a generalized computational experiment, as well as a number of software tools for its implementation were developed in Keldysh Institute of Applied Mathematics RAS. The main aspects of constructing a generalized computational experiment and examples of its implementation are described in detail in [16-23, 28-30].

\section{GENERALIZED COMPUTATIONAL EXPERIMENT}

The emergence of the concept of a generalized computing experiment is associated with the development of high-performance computing clusters and parallel technologies. In problems of computational aerodynamics, parallel technologies usually provide the ability to quickly calculate on detailed grids. However, parallel technologies provide us with another important opportunity. This is the ability to simultaneously calculate on different nodes the same task with different input data. As a rule, such a calculation is performed in multitasking mode. 
This opens up the possibility of implementing a generalized computational experiment. The key parameters of the problem under consideration are divided in certain ranges with a certain step, forming a grid partition of a multidimensional box in a multidimensional space of key parameters. The basic problem is solved using parallel technologies at each point of the grid partition. The obtained results represent multidimensional data volumes. Processing, analysis and visual presentation of this data is carried out using methods of visual analytics and scientific visualization. This computing technology is the most general description of a generalized computing experiment.

Obviously, such a concept can be applied to a wide range of tasks. This range includes parametric studies, optimization problems. A generalized computational experiment is an effective tool for solving inverse problems.

A large number of different applications of a generalized computational experiment are described in detail in [16-23, 28-30]. The concept of a generalized computational experiment was applied to a wide range of both model and practical problems.

These tasks include the analysis of the interaction of a viscous supersonic flow with a jet barrier, the flows in the wake of the body, the problems of the interaction of jets, the problem of flowing around a cone at an angle of attack, the problem of oblique shock waves, and many others. The approach to constructing a generalized computational experiment was applied to the problem of finding the optimal three-dimensional shape of the blades assembly for a power plant in terms of power loads.

Also, this approach was applied to the problems of verification of numerical methods. A comprehensive comparative analysis of a number of solvers of the OpenFOAM open software package [24] was carried out in [20-23, 29, 30]. As basic tasks, we used problems that have a reference solution (exact solution or experimental data). These tasks include the problem of a supersonic inviscid flow around a cone at an angle of attack and the problem of an oblique shock wave formation. In both cases, a class of problems was considered, formed by key parameters variations of the problem in question.

\section{THE APPROACH OF ACCURACY ESTIMATION ON THE ENSEMBLE OF SOLUTIONS}

The estimation of the accuracy of numerical methods in the absence of a reference solution is a separate problem.

Undoubtedly, at present, the understanding of the need to estimate the calculation error is present in the field of CFD and is even formulated as standards [13-15]. However, the methods proposed there are based mainly on the convergence of the solution over the grid (according to [2], this approach goes back to C. Runge) and on Richardson's extrapolation. Both of these approaches are based on the asymptotic behavior of the lowest (in the expansion in terms of the grid step) term of the approximation error and, accordingly, do not provide strict inequalities in the error estimation. For convergence "by adhesion" (Runge), the difference of two solutions (on coarse and fine meshes) is used as an estimate of the error. In Richardson's method, this difference between the solutions is refined using a coefficient that depends on the order of approximation. An additional problem in the field of CFD, which complicates the application of the Richardson method, is the space-variable order of convergence of different algorithms. In particular, on the shock wave for schemes of any approximation order, the convergence order demonstrates values around unity. To take this 
effect into account, the generalized Richardson method is used, which allows one to estimate the local order of convergence. Unfortunately, this method is significantly unstable and requires at least four successive mesh refinements, which creates huge computational problems.

One of the alternatives in this case is the estimation of accuracy on the ensemble of solutions. The ensemble of solutions obtained by various numerical methods on the same grid allows us to estimate the location of the exact solution and to divide the obtained numerical solutions into clusters of different levels of accuracy. This direction is being actively developed at present and is presented in [25-27]. A natural drawback of this approach is the need for researcher to have at his disposal a certain number of solvers that implement numerical methods with different computational properties.

In general, a fairly large volume of numerical experiments [25-27] confirms the possibility of estimating the approximation error on an ensemble of independent numerical calculations, which cannot but arouse interest in the analysis of this approach. It seems likely that the transition from a single numerical solution to an ensemble of independent solutions opens up opportunities for the implementation of non-standard concepts of a numerical solution. These topics need deeper analysis and development. However, if successful, one can hope for the creation of computationally efficient algorithms that ensure the verification of numerical solutions even in the absence of reference solutions. An important role in this can be played by the construction of a generalized computational experiment, where the parameter is the choice of the solver, and the numerical solution is implemented in parallel mode simultaneously for the solvers participating in the calculation. In this case, in the presence of a certain number of independent solvers providing an ensemble of solutions, the construction of a generalized computational experiment can dramatically speed up the estimate.

\section{COMPARATIVE ACCURACY ESTIMATION USING REFERENCE SOLUTION}

This section provides two examples of constructing a generalized computational experiment for a comparative assessment of numerical methods accuracy. As examples, we use the numerical results described in detail in the authors' works [20, 23, 29, 30]. In these papers, two classes of computational gas dynamics problems were considered.

The first class of problem describes a supersonic inviscid flow around a cone at an angle of attack.

The second class describes the incidence of an inviscid supersonic gas flow onto a flat plate at an angle of attack. Both of these problems are fairly well known. The first problem has a tabular solution [36], used as a reference solution. The second problem has an exact solution. We consider the first class of problem for 3D statement. The second class is considered as $2 \mathrm{D}$ problem.

Let's consider the first class of problems. We solve the problem of a supersonic gas flow around a cone at an angle of attack. Variable parameters are angle of attack $\alpha=0^{\circ}, 5^{\circ}, 10^{\circ}$, Mach number $M=3,5,7$ and cone half-angle $\beta=10^{\circ}, 15^{\circ}, 20^{\circ}$. The flow scheme is shown in Figure 1. The conditions of the incoming stream at the input are indicated by the index " $\infty$ ", and at the output, by the index $\xi$, since the solution is self-similar and depends on the dimensionless variable. 
For calculation, the Euler system of equations is used. The system is supplemented by the ideal gas equation of state.

Three solvers were selected from the OpenFOAM software package: rhoCentralFoam $(\mathrm{rCF})$, sonicFoam $(\mathrm{sF})$, and pisoCentralFoam (pCF). Solver rhoCentralFoam is based on a central-upwind scheme which is a combination of central difference and upwind schemes [31,32]. Solver sonicFoam is based on the PISO algorithm (Pressure Implicit with Splitting of Operator) [33]. Solver pisoCentralFoam is a combination of a central-upwind scheme with the PISO algorithm [34]. This solver is not included in the standard set of OpenFOAM solvers. It was created by independent team of developers at the Ivannikov Institute for System Programming RAS. All the calculations were performed using the OpenFOAM version 2.3.0.

We solved the problem with each solver for the entire set of variable parameters. Thus, we obtained a set of numerical solutions. The exact solution was obtained by interpolating the table solution from [3]. Then we found the error of the solution in the norms L1 and L2. Since different solvers implement different numerical methods, the errors were markedly different from each other. The initial and boundary conditions, as well as the settings of the solvers, were set similarly to $[26,28]$.

Fig. 2 shows the steady-state solution for the pressure field obtained by interpolating the tabular solution from [36], cone half-angle $\beta=20^{\circ}$, angle of attack $\alpha=10^{\circ}$, Mach number $\mathrm{M}$ $=3$.

Figure 3 shows the error surface in L2 norm for this problem with the variation of the solver and the half-cone angle at fixed Mach number 3 and fixed angle of attack $5^{\circ}$. It can be seen that the deviation from the exact solution increases with the growth of the half-solution angle. One can also see that the rhoCentralFoam and pisoCentralFoam solvers are approximately equally accurate, while the sonicFoam solver accuracy is much lower.

Figure 4 shows the error surface for the same problem with variation of solvers and angle of attack at a Mach number of 5 and a cone half-angle of $15^{\circ}$.

Figure 5 shows the error surface (deviation from reference solution) for the angle of attack $5^{\circ}$ and a cone half-angle $20^{\circ}$ with variation of solvers and Mach number.

Thus, analyzing Figures 3, 4, 5 we see that for all solvers, the error increases with increasing the angle of attack, the angle of the cone half-angle and Mach number.

So, we have here the accuracy assessment for all three solvers participating in this research. This is the result of constructed generalized numerical experiment for the class of problems in question.

It is easy to see that in this case the numerical result of the generalized computational experiment is the error function in the L2 norm of 4 variables (Mach number, angle of attack, cone half angle, solver number):

$$
\operatorname{Err}=F(M, \alpha, \beta, N s)
$$

A complex visual representation of such a function is a separate task and is not considered in this article.

For the considered class of problems, the construction of a generalized computational experiment provided a full-fledged comparative estimate of the accuracy of the three selected solvers of the OpenFOAM package in the range of variation of the determining parameters. 


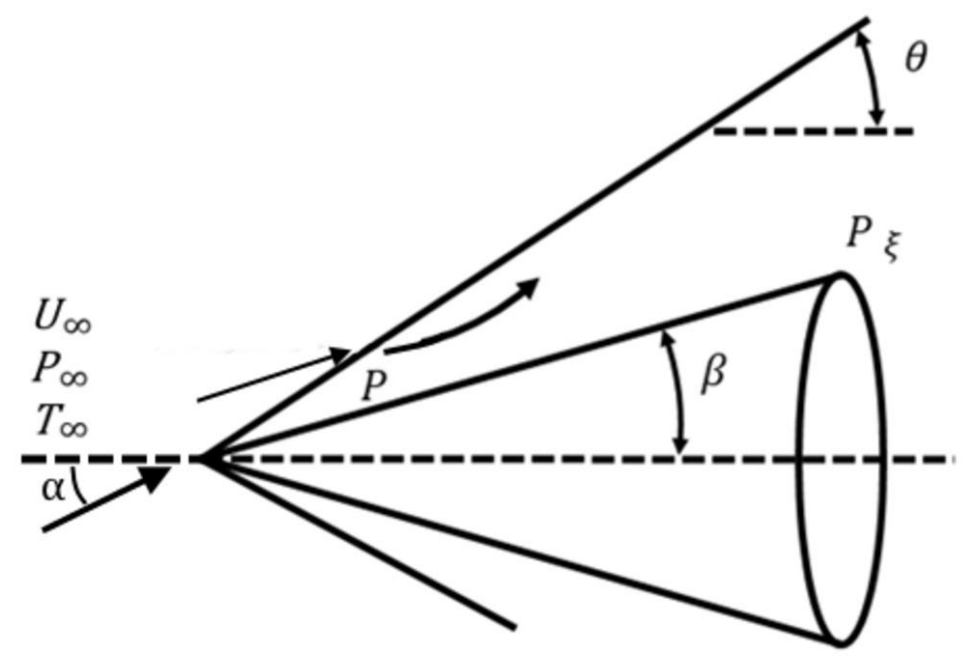

Fig. 1. Flow scheme.
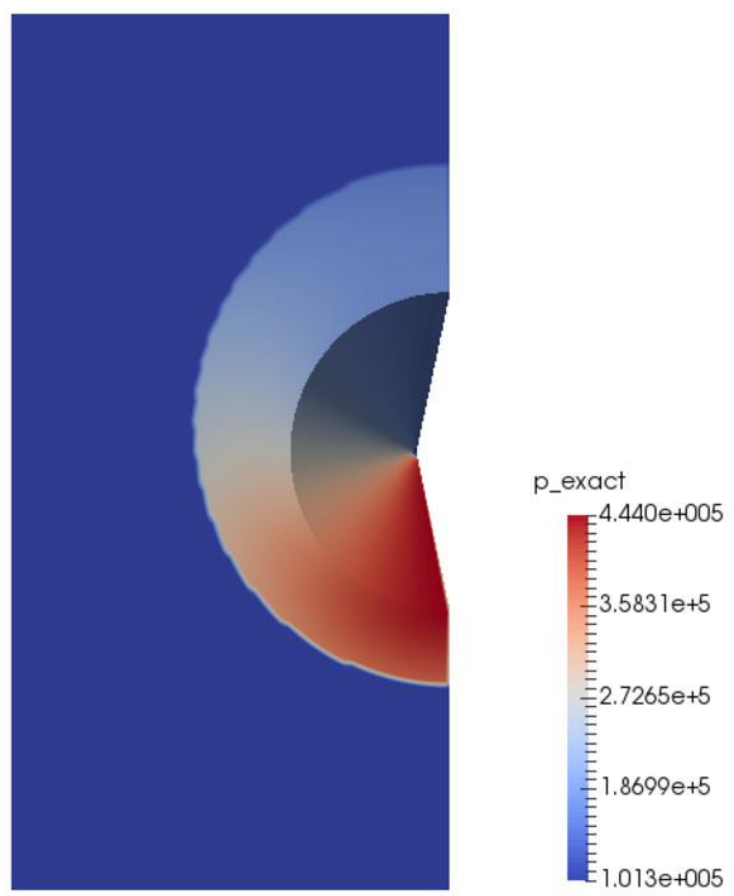

Fig. 2. Pressure field for steady flow. 


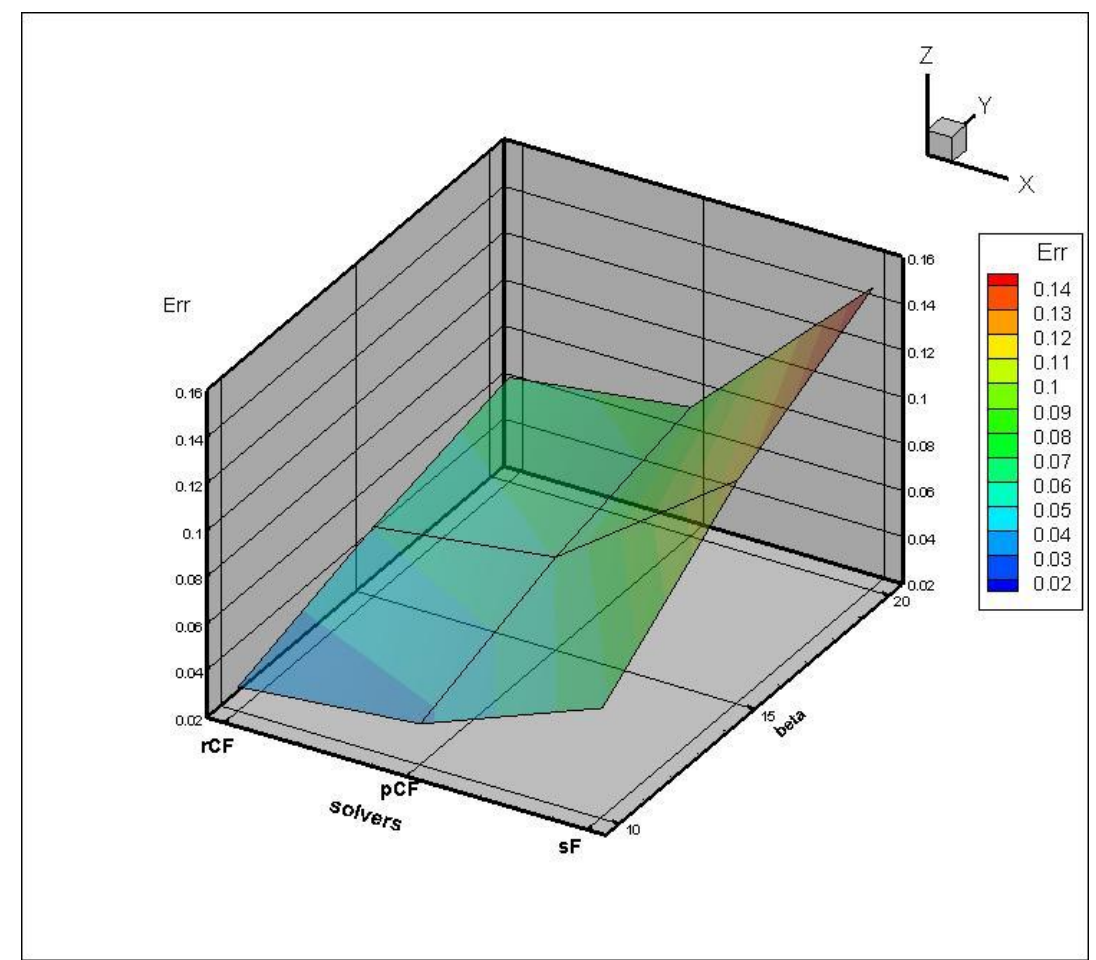

Fig. 3. Image of the error surface for the Mach number 3 and the angle of attack $5^{\circ}$ with variation of solvers and half-cone angle.

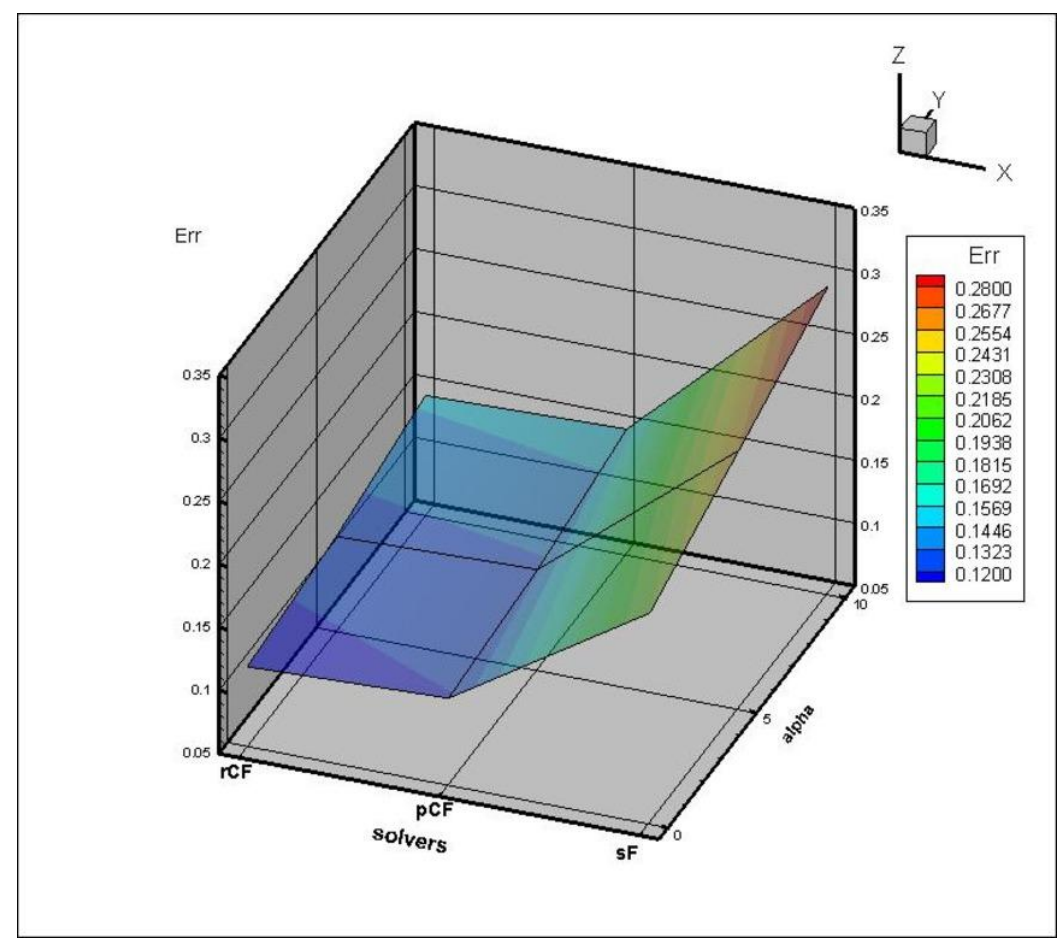

Fig. 4. Image of the error surface for the Mach number 5 and a cone half-angle $15^{\circ}$ with variation of solvers and angle of attack. 


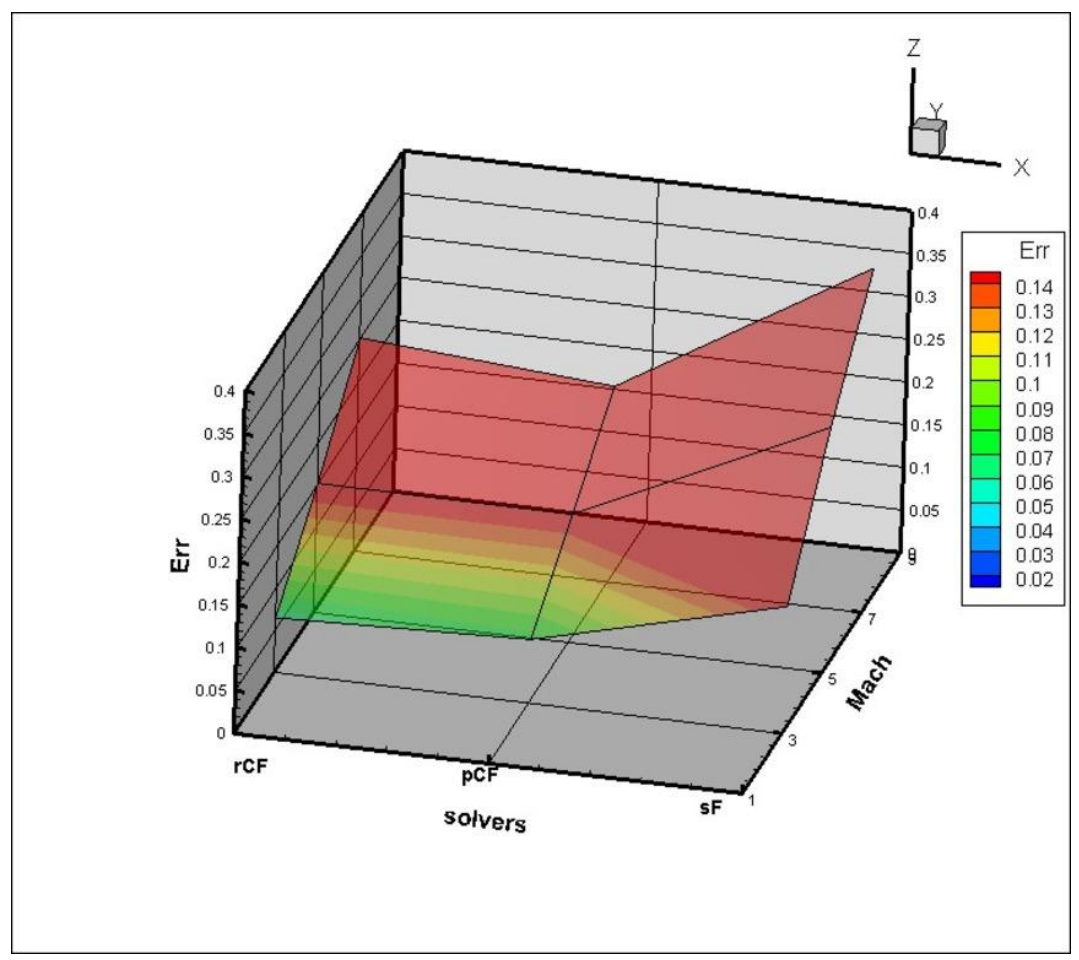

Fig. 5. Image of the error surface for the angle of attack $5^{\circ}$ and a cone half-angle $20^{\circ}$ with variation of solvers and Mach number.

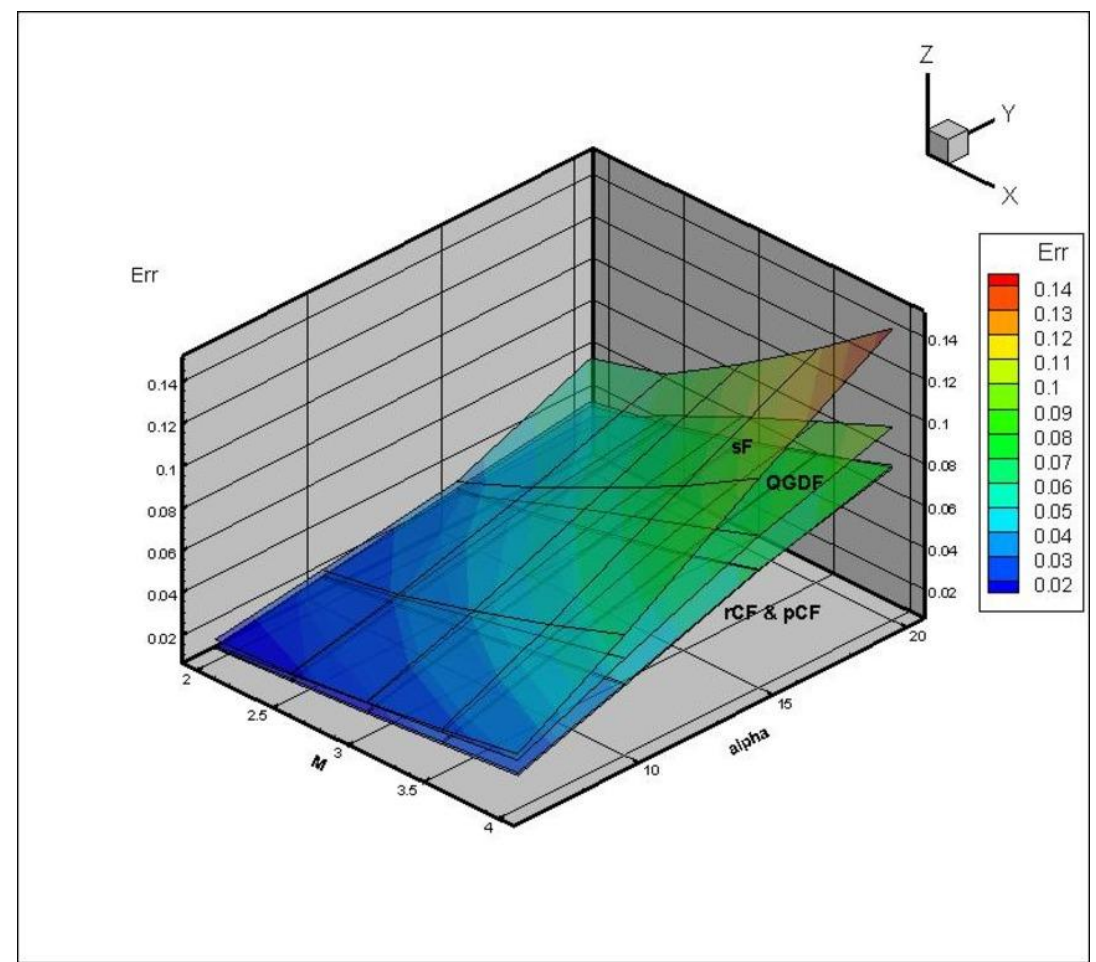

Fig. 6. Error surfaces with variation of the Mach number and angle of attack for the oblique shock wave [29]. 
Let's consider another class of problems. In this case we use well-known problem of oblique shock wave formation. We consider this problem in 2D statement.

A supersonic gas flow falls on the plate at an angle. Reflecting from the plate, the flow forms an oblique shock wave. The problem has exact solution. In the problem, the Mach number $M$ and the angle of incidence of the supersonic flow $\beta$ varied, similarly to [29,30]. Figure 6 shows the error surfaces for this problem for 4 solvers: rhoCentralFoam (rCF), sonicFoam ( $\mathrm{sF}$ ), pisoCentralFoam (pCF), QGDFoam (QGDF). Now we include into consideration a new solver QGDFoam (QGDF). This solver is based on a system of quasihydrodynamic equations. The solver was also created by independent developers [35].

Carrying out similar calculations for several numerical methods implemented in the solvers of the open software package OpenFOAM, makes it possible to build several such surfaces on one drawing. This opens up the possibility of a deep and clear comparative analysis of the accuracy of the studied numerical methods. The construction of such a generalized computational experiment involves the creation of computational technology from solving a direct problem up to visual analysis of the results.

This technique allows carrying out a detailed visual comparison of deviations from the exact solution. It can be seen that in our case, all error surfaces change in the same way. The error increases with the growth of key parameters. The best accuracy in this class of problems is provided by the $\mathrm{rCF}$ and $\mathrm{pCF}$ solvers, for which the error surfaces are almost identical. Thus, the construction of a generalized computational experiment allows us to conduct a fullfledged comparative accuracy assessment for four solvers of the OpenFOAM software package in the class of problems. The class of tasks in this particular case is determined by the basic task (oblique shock wave) and the ranges of variation of the key parameters of the problem - the Mach number and angle of attack.

The use of a generalized computational experiment makes it possible to involve new numerical methods in research on the comparative assessment of accuracy. This problem was also solved for the numerical WW-method [37], which does not belong to OpenFOAM solvers, but is implemented as a separate software package. It's ADI-method [38] modification using hybrid implicit finite difference scheme. The review describing hybrid schemes is presented in [39]. The scheme [37] has second order accuracy in space and time. Also the scheme (we'll call this scheme as WW-scheme) is unconditionally stable and simple for programming. Except these properties WW-scheme has one interesting and useful feature. When non-linear problem with strong shocks is solved, one has to reduce undesirable solution oscillations. There are two ways for this. The first way is concerned with procedure of smoothing between time-steps. The second way consists in adding some terms with artificial viscosity to basic equations. Both ways require more calculations and complicate algorhytm. The present numerical method doesn't need these ways. Needed for stabilization of solution artificial viscosity is an internal property of WW-scheme. One can regulate the artificial viscosity by the choice of weight parameters. This property is quite suitable for practical applications. It should be noted that QGDFoam solver [35] also has regulated artificial viscosity. This opens up prospects in the future for studying the effect of artificial viscosity on the comparative assessment of accuracy for similar numerical methods. 


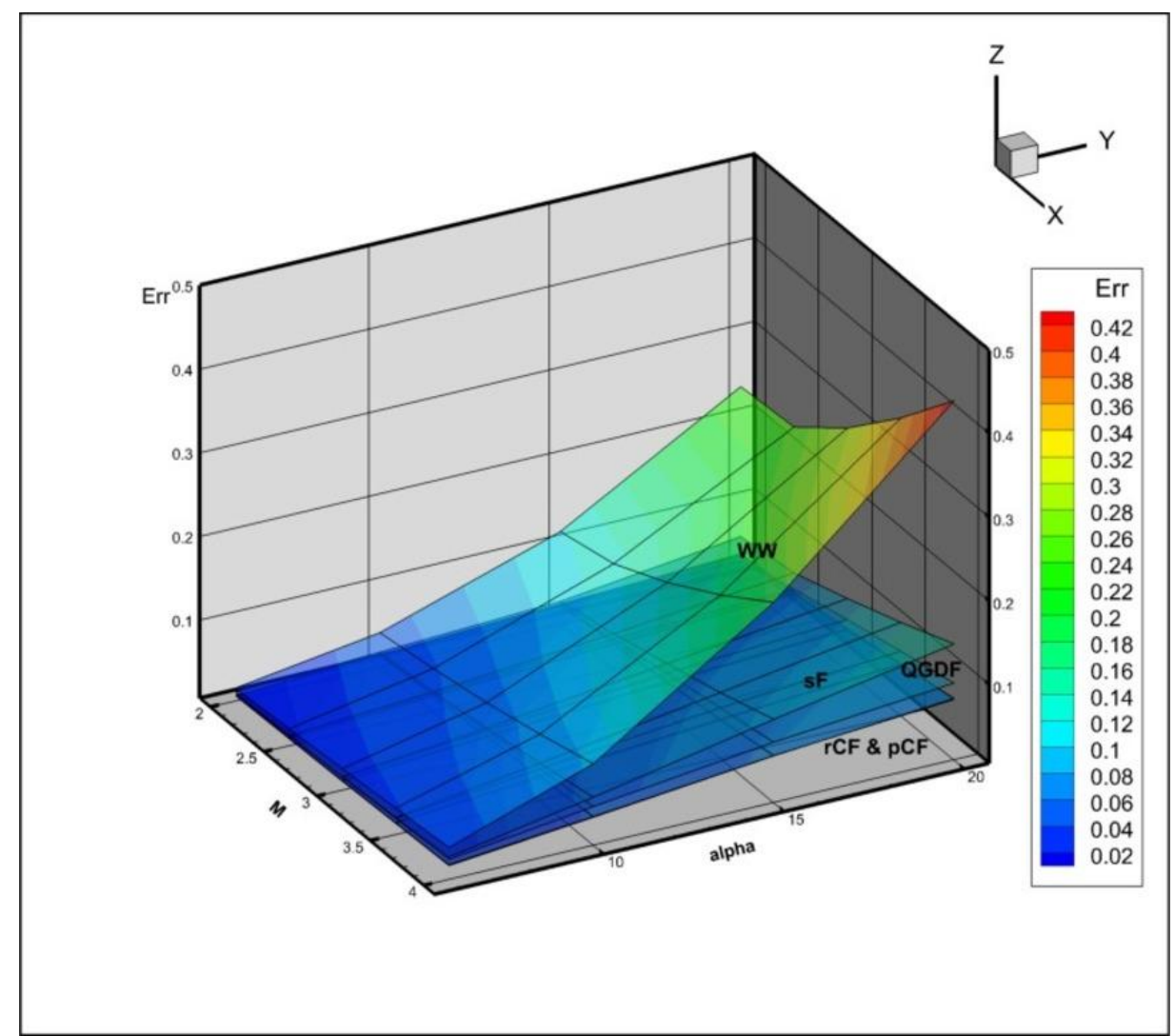

Fig. 7. Error surfaces with variation of the Mach number and angle of attack for the oblique shock wave for OpenFOAM solvers and WW-method.

Figure 7 presents the error surfaces for OpenFOAM solvers with addition such surface for WW-method. One can see that for WW-method, the error increases with an increase in the Mach number and the angle. You can also notice that the error surface for the WW-method is located much higher than the similar surfaces for OpenFOAM solvers. However, this discrepancy can presumably be reduced using artificial viscosity parameters.

The images of error surfaces presented in Figures 6, 7 give a fairly clear idea of the comparative accuracy of numerical methods in the class of problems. However, for a more complete assessment, we enter an integral characteristic for each surface. We will call this characteristic the Error Index (EI). The error index is defined as follows.

Let we have $K$ key parameters. Each of them has its own grid partition, and $A$ - the deviation from the exact solution at each point of the grid partition. We denote the total number of points in the resulting multidimensional space as $N$. Then the error index is defined as:

$$
E I=\left(\sum A\right) / N
$$


First, we calculate the Error Index for the problem of flow around a cone at an angle of attack.

\begin{tabular}{|l|l|l|l|}
\hline Solver & rCF & pCF & sF \\
\hline Error Index & 0.13336 & 0.13366 & 0.24086 \\
\hline
\end{tabular}

Table 1. Error Index values for the problem of flow around a cone at an angle of attack

Next, we calculate the values of the error index for the problem of oblique shock formation.

\begin{tabular}{|l|c|c|c|c|c|}
\hline Solver & rCF & pCF & QGDF & sF & WW \\
\hline Error Index & 0.037734 & 0.038751 & 0.0453406 & 0.058216 & 0.14888 \\
\hline
\end{tabular}

Table 2. Error Index values for the problem of oblique shock formation

Tables 1 and 2 show that the values of the error index EI fully correspond to the relative positions of the numerical results presented in figures above. Therefore, the calculated error index can serve as a characteristic of the accuracy of numerical methods in the selected class of problems.

\section{CONCLUSIONS}

The application of a generalized computational experiment to the problems of comparative estimation of the accuracy of numerical methods is considered. A generalized computational experiment allows simultaneous calculations of the same problem with different input data based on parallel technologies in a multitasking mode. The obtained multidimensional results are examined using visual analysis tools.

Two examples of constructing a generalized computational experiment for classes of problems are presented - flow around a cone at an angle of attack and the formation of an oblique shock wave. For both cases the class of problems is formed on the basis of the basic problem and variations of the determining parameters of the problem. For both classes of problems, a comparative assessment of the accuracy of the solvers of the software package OpenFOAM. For the case of oblique shock wave WW-method is added to comparison. This method does not belong to OpenFOAM solvers and is implemented as a separate software package. An example of constructing error surfaces is given. The concept of a numerical method error index for a class of problems is introduced. Such indexes are computed for all cases in this research. The calculated error indexes can serve as a characteristic of the accuracy of numerical methods in the selected class of problems.

The construction of a generalized computational experiment can serve as an effective tool for verification problems. 


\section{REFERENCES}

[1] R.D. Skeel, "Thirteen ways to estimate global error", Numer. Math, 48, 1-20 (1986).

[2] S.I. Repin, A posteriori estimates for partial differential equations, Walter de Gruyter, Vol. 4 (2008).

[3] J.T. Oden and S. Prudhomme, "Goal-oriented error estimation and adaptivity for the finite element method", Comput. Math. Appl., 41, 735-756 (2001).

[4] S. Prudhomme and J.T. Oden, "On goal-oriented error estimation for elliptic problems: application to the control of pointwise errors", Comput. Method. Appl. M., 176, 313-331 (1999).

[5] M Ainsworth and J. T. Oden, A posteriori error estimation in finite element analysis, New York: Wiley (2000).

[6] I. Babuska, J. Osborn, "Can a finite element method perform arbitrarily badly?", Math. Comput., 69, 443-462 (2000).

[7] M.H. Carpenter and J.H. Casper, Accuracy of shock capturing in two spatial dimensions, AIAA J., 37, 1072-1079 (1999).

[8] J.W. Banks, J.A.F. Hittinger and C.S. Woodward, "Numerical error estimation for nonlinear hyperbolic PDEs via nonlinear error transport", Comput. Method. Appl. M., 213, 1-15 (2012).

[9] F. Rauser, J. Marotzke and P. Korn, "Ensemble-type numerical uncertainty quantification from single model integrations", J. Comp. Phys., 292, 30-42 (2015).

[10] C. Johnson, "On computability and error control in CFD", Int. J. Numer. Meth. Fl., 20, 777788 (1995).

[11] I. Babuska and W. Rheinboldt, "A posteriori error estimates for the finite element method", Int. J. Numer. Meth. Eng., 12, 1597-1615 (1978).

[12] Ch.J. Roy and A. Raju, "Estimation of discretization errors using the method of nearby problems", AIAA J., 45, 1232-1243 (2007).

[13] Guide for the verification and validation of computational fluid dynamics simulations, American Institute of Aeronautics and Astronautics, AIAA-G-077-1998, Reston, VA (1998).

[14] Standard for verification and validation in computational fluid dynamics and heat transfer, ASME V\&V 20-2009 (2009).

[15] Federal standard P 57700.12-2018. Numerical simulation of supersonic flows for an inviscid gas. Software verification - National standard of the Russian Federation for numerical modeling of physical processes (2018).

[16] A.E. Bondarev, "Analysis of space-time flow structures by optimization and visualization methods", Lect. Notes Comput. Sc., 7870, 158-168 (2013).

[17] A.E. Bondarev and V.A. Galaktionov, "Parametric optimizing analysis of unsteady structures and visualization of multidimensional data", Int. J. Model. Simul. Sci. Comput., 04 No. supp01 (2013).

[18] A.E. Bondarev, "On the Construction of the Generalized Numerical Experiment in Fluid Dynamics", Mathematica Montisnigri, 42, 52-64 (2018).

[19] A.E. Bondarev, "On visualization problems in a generalized computational experiment", Scientific Visualization, 11,156-162 (2019).

[20] A.E. Bondarev and A.E. Kuvshinnikov, "Analysis of the accuracy of OpenFOAM solvers for the problem of supersonic flow around a cone", Lect. Notes Comput. Sc., 10862, 221-230 (2018).

[21] A.E. Bondarev, "On the estimation of the accuracy of numerical solutions in CFD problems", Lect. Notes Comput. Sc., 11540, 325-333 (2019).

[22] A.E. Bondarev and V.A. Galaktionov, "Generalized computational experiment and visual analysis of multidimensional data", Scientific Visualization, 11, 102-114 (2019). 
[23] A.E. Bondarev, A.E. Kuvshinnikov, "Analysis of the accuracy of OpenFOAM solvers for the problem of supersonic flow around a cone", Lect. Notes Comput. Sc., 10862, 221-230 (2018).

[24] OpenFOAM Foundation. https://openfoam.org (Accessed August 5, 2020).

[25] A.K. Alekseev and A.E. Bondarev, "On exact solution enclosure on ensemble of numerical simulations", Mathematica Montisnigri, 38, 63-77 (2017).

[26] A.K. Alekseev, A.E. Bondarev and A.E. Kuvshinnikov, "Verification on the ensemble of independent numerical solutions", Lect. Notes Comput. Sc., 11540, 315-324 (2019).

[27] A.K. Alekseev and A.E. Bondarev, "Estimation of the distance between true and numerical solutions", Comput. Math. Math. Phys., 59, 857-863 (2019).

[28] A.K. Alekseev, A.E. Bondarev and A.E. Kuvshinnikov, "On uncertainty quantification via the ensemble of independent numerical solutions", J. Comput. Sci., 42, 101114 (2020).

[29] A.K. Alekseev, A.E. Bondarev and A.E. Kuvshinnikov, "Comparative analysis of the accuracy of OpenFOAM solvers for the oblique shock wave problem", Matematica Montisnigri, vol. 45, 95-105 (2019).

[30] A. Bondarev, A. Kuvshinnikov, "Parametric study of the accuracy of OpenFOAM solvers for the oblique shock wave problem", IEEE The Proceedings of the 2019 Ivannikov ISPRAS Open Conference, 108-112 (2019).

[31] A. Kurganov and E. Tadmor, "New high-resolution central schemes for nonlinear conservation laws and convection-diffusion equations", J. Comput. Phys., 160, 241-282 (2000).

[32] C. Greenshields, H. Wellerr, L. Gasparini, and J. Reese, " Implementation of semi-discrete, non-staggered central schemes in a colocated, polyhedral, finite volume framework, for highspeed viscous flows", Int. J. Numer. Meth. Fluids, 63, 1-21 (2010).

[33] R. Issa, "Solution of the implicit discretized fluid flow equations by operator splitting", $J$. Comput. Phys., 62, 40-65 (1986).

[34] M. Kraposhin, A. Bovtrikova and S. Strijhak, "Adaptation of Kurganov-Tadmor numerical scheme for applying in combination with the PISO method in numerical simulation of flows in a wide range of Mach numbers", Procedia Computer Science, 66, 43-52 (2015).

[35] M.V. Kraposhin, E.V. Smirnova, T.G. Elizarova, and M.A. Istomina, "Development of a new OpenFOAM solver using regularized gas dynamic equations", Computers \& Fluids, 166, 163175 (2018).

[36] K.I. Babenko, G.P. Voskresenskii, A.N. Lyubimov, and V.V. Rusanov, Three-dimensional ideal gas flow past smooth bodies, Moscow: Nauka (1964).

[37] A.E. Bondarev, "On hybrid numerical method for 2d viscous flows", Mathematica Montisnigri, 29, 59-67 (2014).

[38] D.W. Peaceman and H.H. Rachford Jr., "The numerical solution of parabolic and elliptic differential equations", Journal of the Society for Industrial and Applied Mathematics, 3, 28-41 (1955).

[39] A.G. Kulikovsky, N.V. Pogorelov, and A.Yu. Semenov, Matematicheskie voprosy chislennogo resheniya giperbolicheskih sistem uravneniy Moscow, PhysMathLit (2001).

Received June 12, 2020 